\title{
A VLBI view on the extreme scintillator J1819+3845
}

\section{Giuseppe Cimo*}

JIVE

E-mail: cimo@jive.nI

\section{Leonid Gurvits}

JIVE

Delft University of Technology.

E-mail: lgurvits@jive.nI

\section{Preeti Kharb ${ }^{\dagger}$}

Indian Institute of Astrophysics

E-mail: kharbeiiap.res.in

\section{Ger de Bruyn}

ASTRON

E-mail: bruyn@astron.nl

\section{Jean-Pierre Macquart}

Curtin University of Technology

E-mail: J.Macquart@curtin.edu.au

Scintillation of flat spectrum radio sources provides a unique instrument to study the characteristics of the electron density distribution throughout the interstellar medium as well as to study the microarcsecond structure of compact radio objects. During the course of its extreme activity, the quasar J1819+3845 has been observed with a number of VLBI arrays. The fast variability, up to $600 \%$ in less than one hour, has made it very difficult to image the source. The variability has now stopped and we will present scintillation-free VLBI images of J1819+3548 taken with the EVN (2008) and VLBA (2007). We will compare the quiescent phase of J1819+3845 with images of the source during its extreme variability (observed with global and space VLBI). A description of the possible scattering screen and implications for the source internal structure will be presented.

11th European VLBI Network Symposium \& Users Meeting,

October 9-12, 2012

Bordeaux, France

\footnotetext{
* Speaker.

${ }^{\dagger}$ Helena Kluyver visitor at JIVE.
} 


\section{Introduction}

IntraDay Variable sources (IDV) are flat spectrum extragalactic synchrotron sources, often associated with quasars, BL Lacs or radio galaxies, that show flux density variability on time scales shorter than a day (Kedziora-Chudczer et al. 1997 [5]). They are typically unresolved with the longest VLBI baselines at centimetre wavelengths. These sources show intrinsic variations on much longer time scales (months) that sometimes are associated with milliarcsecond structure that evolves on time scales of years (Krichbaum et al. 1997 [6]). However, in case of intrinsic rapid variability, the short time scale sets a limit on the size of the emitting region and allows one to estimate its brightness temperature. The latter would exceed $10^{21} \mathrm{~K}$. Even a highly relativistic jet beamed and doppler boosted toward the line of sight cannot explain such an extreme violation of the Inverse Compton limit $\left(10^{12} \mathrm{~K}\right)$. Therefore, the easiest way to explain the intraday variability is as a propagation effect, similar to that in low frequency variables, due to scintillation in an interstellar screen of ionized gas (Rickett et al. 2001 [7] and references therein). This theory was confirmed by Dennett-Thorpe \& de Bruyn (2002) [3] who monitored flux density variations in J1819+3845 at the VLA and WSRT simultaneously. The light curves from the two telescopes show nearly perfect correlation, but with a time delay of a few minutes, corresponding to the separation of the two telescopes in their motion through the refraction pattern. The quasar J1819+3845 has shown extreme variability with flux density variations in the radio regime up to $600 \%$ in less than one hour (Dennett-Thorpe \& de Bruyn 2002 [3]). The extreme variability of J1819+3548 has been observed since its first observation in 1999 and it has continued for more than 8 years until 2006. Observation taken with VLBA in 2007 and with EVN in 2008 (Cimò et al. 2008 [1]) have shown that the source scintiallation has entered in a quiescent phase and the rapid variability has stopped.

\section{Observations}

The extreme variability of $\mathrm{J} 1819+3845$ has been proven to be due to scattering in the Interstellar medium by a number of different experiments. Such an explanation requires a closeby scattering screen (Dennett-Thorpe \& de Bruyn 2002 [3] and Dennett-Thorpe \& de Bruyn 2003 [4]). Due to its high brightness temperature and its compact nature, J1819+3845 was expected to expand quenching the scintillation or at least changing its variability characteristics following its internal structural variations. However, the variations have been observed continuously along the years showing also a clear annual modulation due to relative motion of the Earth and the scattering screen.

A number of VLBI campaigns have been carried out to image the innermost jet structures of J1819+3845. Three global VLBI experiments, including a Space VLBI epoch, have been carried out while the source was scintillating. The source was later observed with the VLBA and the EVN arrays during its quiescent phase.

The main issue with imaging $\mathrm{J} 1819+3845$ has been its extreme variability. The data reduction is compromised by the difficulties in dealing with phase fluctuations and scintillation. The interstellar scattering covers the structure of the source due to the scattering broadening of the VLBI image. In this paper, we present images of J1819+3845 observed with global VLBI, VLBA and EVN. The Global VLBI observations are part of an experiment carried out at $4.9 \mathrm{GHz}$ in April and 
September 2001 during a space VLBI (VSOP) campaign. The space baselines, however, have not been included in the maps presented here. In February 2007, J1819+3845 has been observed at $8.4 \mathrm{GHz}$ with the VLBA and the Green Bank Telescope. These observations though were effected by bad weather at the GBT and at the telescopes that provided the longest baselines. In March 2008, new observations with the EVN at $4.8 \mathrm{GHz}$ have been carried out. Unfortunately bad weather conditions affected most of the antennas. In particular the 76-meter antenna in Jodrell Bank missed all the experiment due to high wind. Furthermore the station in Shanghai, which had to provide the longest baselines, had severe problems with the recording system.

\section{VLBI maps}

The 2001 Global VLBI observations of J1819+3548 do not present any clear indication for any structural changes with time (see fig. 1). However our imaging process is limited by the scintillation. Analysis of the VLBI structure of the source during its extreme scintillation phase is in progress. For the present work, the global VLBI dataset have been divided in small time intervals in order to minimize the effects of the scintillation. The dataset observed in September 2001 shows less 'scintles' in the light curve because of annual modulation of the scintillation. However, no clear evidence for structure can be found in both the April and September dataset.

In 2007 and 2008, observations of J1819+3548 (Cimò et al 2008 [1]) have shown no signs of any variability. A possible explanation for the cessation of the fast variations in J1819+3548 is that the scattering screen has moved away from our line of sight. On the other hand, the disappearance of the extreme scintillations on J1819+3845 could be due to variations in the source structure at $\mu$-arcsecond scale. A new component, which expands as it evolves would lead to episodic scintillations. The VLBA and EVN data have shown however that the source is unresolved at continental baselines. The lack of variability gave us the possibility to image the source for the first time without seeing it through the ISM turbulent screen. The VLBA and EVN images are shown in figure 2. These maps still show an unresolved J1819+3845. This may be the consequence of the poor resolution due to the lack of the most long baselines. Standard software packages, AIPS and Difmap, have been used for the data reduction. Further analysis is in progress to take into account the variability in the VLBI data and to include the VSOP baselines (Cimò et al in preparation).

\section{The Interstellar Medium}

Episodic variability is a common phenomenon in flat spectrum radio sources. In some cases, long-term (within months or years) changes in the IDV pattern can be due to source-intrinsic effect, such as the emission of new components quenching the scintillation. A source scintillates as long as its angular size is smaller than the Fresnel scale of the ISM perturbation in our Galaxy. The scintillations would stop once the component expands exceeding the Fresnel size. The disappearance of the extreme scintillations on J1819+3845 could be due to variations in the source structure at $\mu$-arcsecond scale. A new component, which expands as it evolves would lead to episodic scintillations. On the other hand, evidences for a new component such as strong changes in flux density have not been confirmed by the observations. Finally, assuming a new component emitted from the core has emerged, we used the EVN data to see if this component is resolved at milliarcsecond 

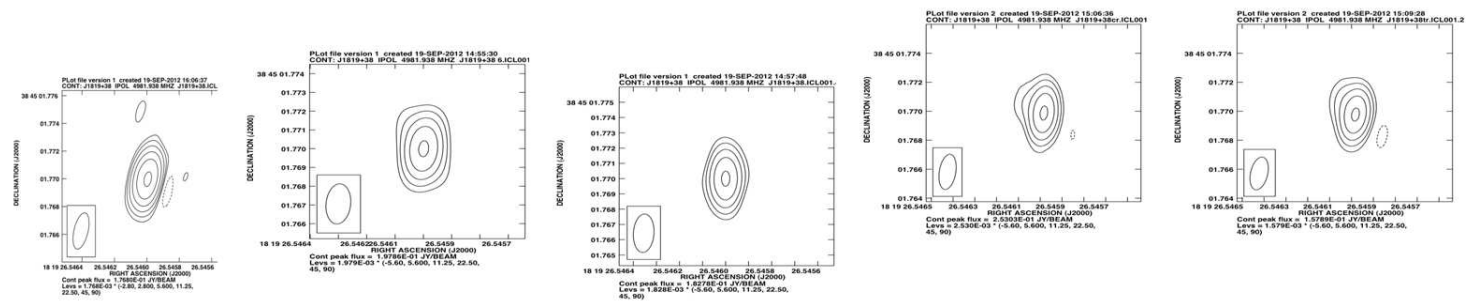

Figure 1: A series of global VLBI snapshots of J1819+3845. The snapshots were selected to minimize the effects of variability. The resulting images do not show significant structure.

scale a year after the last recorded variability. However, the source appears unresolved on all EVN baselines.

A change in the scattering material along the line of sight to J1819+3845 is another possible (and maybe more plausible) explanation. We can assume that the screen has simply moved away, and therefore we can immediately put some constraints on the size of the scattering material. Assuming a typical velocity of the cloud of about $35 \mathrm{~km} / \mathrm{s}$, the screen moved of $5.2 \mathrm{AU}$ in less than a year. Since we do not know when the scintillation started, we can not define the linear size of the screen. As a limit, we can evaluate a lower limit for the extent of the scattering screen in the direction of the transverse velocity of about $60 \mathrm{AU}$. For a more detailed analysis see de Bruyn \& Macquart (2012) [2].
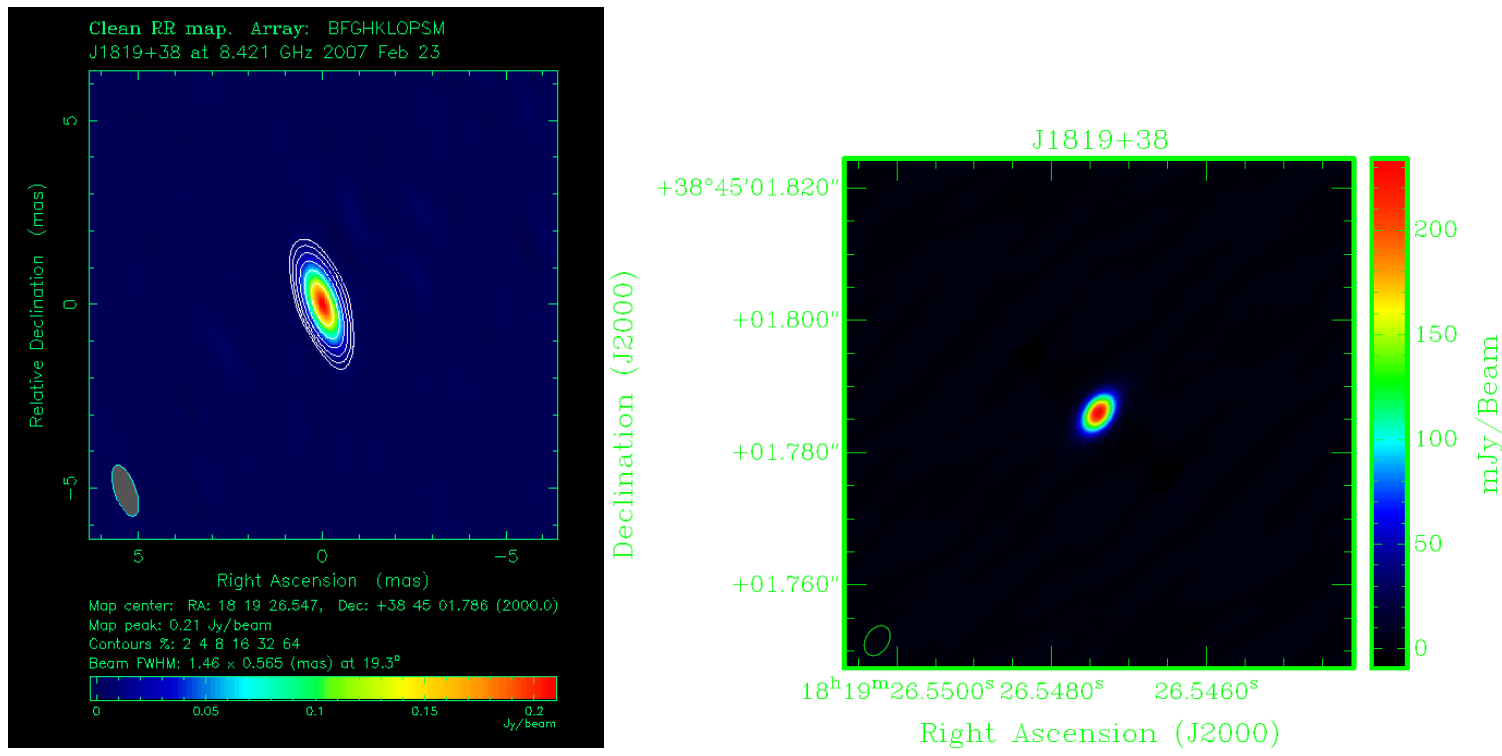

Figure 2: Left: VLBA map of J1819+3845 on February 2007. Right: EVN map of the source observed on March 2008. 


\section{Conclusions}

A number of VLBI arrays have been used to image the scintillator J1819+3845. The extreme variability of the source has been observed for more than 8 years and the VLBA experiment in 2007 has shown that the variability has stopped. The lack of variability has made possible to image the source. After a year from the last observed scintillation, the source is unresolved at VLBI scale.

Local ISM changes (the screen moved away) can explain the end of the extreme scintillation of J1819+3845. Taking into account previous IDV measurments of this quasar, it has been possible to put a lower limit of $60 \mathrm{AU}$ to the size of the cloud responsible of the previously observed extreme variations.

\section{References}

[1] G. Cimò and L. Gurvits 2008, Two-Dimensional Time Delay Measurement of a fast Scintillator using VLBI arrays., 2008. PoS: Proceedings of the 9th European VLBI Network Symposium. (p.13).

[2] G. de Bruyn \& J.-P. Macquart, 2012, The intra-hour variable quasar J1819+3845: 13-year evolution, jetpolarization structure and interstellar scattering screen properties, $A \& A$

[3] J. Dennett-Thorpe \& A. G. de Bruyn, 2002, Interstellar scintillation as the origin of the rapid radio variability of the quasar J1819+3845, Nature 415 (57).

[4] J. Dennett-Thorpe \& de A. G. Bruyn, 2003, Annual modulation in the scattering of J1819+3845: Peculiar plasma velocity and anisotropy, A\&A 404 (113).

[5] L. Kedziora-Chudczer, D. L. Jauncey, M. H. Wieringa, M. A. Walker, et al. 1997, PKS 0405-385: The Smallest Radio Quasar?, Ap. J. 490 (L9).

[6] T. P. Krichbaum, D. A. Graham, A. Greve, J. E. Wink, et al. 1997, 215 GHz VLBI observations of bright Active Galactic Nuclei, A\&A $\mathbf{3 2 3}$ (17).

[7] B. J. Rickett, A. Witzel, A. Kraus, T. P. Krichbaum \& S. J. Qian, 2001, Annual Modulation in the Intraday Variability of Quasar 0917+624 due to Interstellar Scintillation, Ap. J. 550 (L11). 